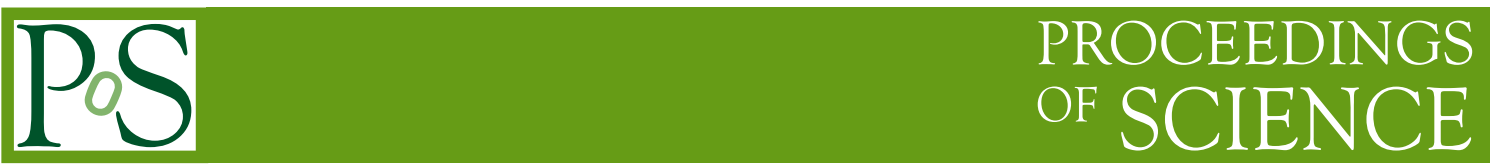

\title{
The curvature of the QCD crossover line
}

\author{
Patrick Steinbrecher (for the HotQCD collaboration) \\ Fakultät für Physik, Universität Bielefeld, D-33615 Bielefeld, Germany \\ Physics Department, Brookhaven National Laboratory, Upton, NY 11973 \\ E-mail: psteinbrecherabnl.gov
}

\begin{abstract}
We map out the QCD crossover line $\frac{T_{c}\left(\mu_{B}\right)}{T_{c}(0)}=1-\kappa_{2}\left(\frac{\mu_{B}}{T_{c}(0)}\right)^{2}-\kappa_{4}\left(\frac{\mu_{B}}{T_{c}(0)}\right)^{4}+\mathscr{O}\left(\mu_{B}^{6}\right)$ for the first time up to $\mathscr{O}\left(\mu_{B}^{4}\right)$ for a strangeness neutral system by performing a Taylor expansion of chiral observables in temperature $T$ and chemical potentials $\mu$. At vanishing chemical potential, we report a crossover temperature $T_{c}(0)=(156.5 \pm 1.5) \mathrm{MeV}$ defined by the average of several chiral susceptibilities. For a system with thermal conditions appropriate for a heavy-ion collision, we determined a curvature from the subtracted condensate as $\kappa_{2}=0.0120(20)$ and from the disconnected susceptibility as $\kappa_{2}=0.0123(30)$. The next order $\kappa_{4}$ is significantly smaller. We also report the crossover temperature as a function of the chemical potentials for: baryon-number, electric charge, strangeness and isospin. Additionally, we find that $T_{c}\left(\mu_{B}\right)$ is in agreement with lines of constant energy density and constant entropy density. Along this crossover line, we study net baryon-number fluctuations and show that their increase is substantially smaller compared to that obtained in HRG model calculations. Similarly, we analyze chiral susceptibility fluctuations along the crossover line and show that these are constant. We conclude that no signs for a narrowing of the crossover region can be found for baryon chemical potential $\mu_{B}<250 \mathrm{MeV}$.
\end{abstract}

The 36th Annual International Symposium on Lattice Field Theory - LATTICE2018

22-28 July, 2018

Michigan State University, East Lansing, Michigan, USA. 


\section{Introduction}

We present results from our study of the crossover of quantum chromodynamics (QCD) in (2+1)-flavor QCD as a function of the baryon chemical potential $\mu_{B}$. We base this analysis on findings $[1,2]$ that at vanishing chemical potentials strong interaction matter does not have a genuine phase transition from a gas of hadrons and their resonances (HRG) to a quark-gluon plasma (QGP). Our goal is to understand up to which baryon chemical potential the crossover is still analytic. In other words, we are searching for signs of a second order QCD critical point which would be the start of a genuine first order phase transition line. In the following, we consider chiral observables as the crossover and a possible phase transition are supposed to be closely related to chiral symmetry restoration. Particularly important are the subtracted chiral condensate

$$
\Sigma_{\text {sub }} \equiv m_{s}\left(\Sigma_{u}+\Sigma_{d}\right)-\left(m_{u}+m_{d}\right) \Sigma_{s} \quad \text { with } \quad \Sigma_{f}=\frac{T}{V} \frac{\partial}{\partial m_{f}} \ln Z,
$$

the subtracted and disconnected chiral susceptibility

$$
\chi_{\text {sub }} \equiv \frac{T}{V} m_{s}\left(\frac{\partial}{\partial m_{u}}+\frac{\partial}{\partial m_{d}}\right) \Sigma_{\text {sub }}, \quad \chi_{\text {disc }}=m_{s}^{2}\left(\chi_{u, \text { disc }}+\chi_{d, \text { disc }}+2 \chi_{u d}\right)
$$

with

$$
\chi_{f g}=\frac{\partial}{\partial m_{f}} \Sigma_{g} \quad \text { and } \quad \chi_{\mathrm{f}, \mathrm{disc}}=\frac{T}{V} \frac{1}{16}\left[\left\langle\left(\operatorname{Tr} M_{f}^{-1}\right)^{2}\right\rangle-\left\langle\operatorname{Tr} M_{f}^{-1}\right\rangle^{2}\right] .
$$

Here, $M_{f}$ is the fermion matrix for quark flavor $f$. The observables are shown in Fig. 1. Their Taylor expansions in chemical potentials have been described in [3]. If a critical point exists, we should be able to observe scaling with the critical exponents of a three-dimensional Ising model at finite baryon chemical potential. When approaching a critical point, a significant increase of chiral susceptibility fluctuations along the crossover must be observed. We have generated gauge field ensembles using a RHMC for 4 lattice volumes with $N_{\tau}=6,8,12$ and 16 in a temperature range from $135 \mathrm{MeV}$ to $175 \mathrm{MeV}$. The simulations have been performed using the tree-level improved HISQ formulation with two degenerate light quarks and a heavier strange quark set to their physical values corresponding to a pion mass of about $138 \mathrm{MeV}$. The scale has been set using the kaon decay constant [4].

\section{The QCD crossover line}

The crossover line can be parameterized as

$$
\frac{T_{c}\left(\mu_{B}\right)}{T_{0}}=1-\kappa_{2}\left(\frac{\mu_{B}}{T_{0}}\right)^{2}-\kappa_{4}\left(\frac{\mu_{B}}{T_{0}}\right)^{4}+\mathscr{O}\left(\mu_{B}^{6}\right),
$$

where $T_{0}$ is the crossover temperature at zero chemical potential given by so-called pseudo-critical temperatures. Their continuum extrapolations are shown in Fig. 1 (right). In the continuum, all considered pseudo-critical temperatures converge to similar values. This is why we quote a combined value of $T_{0}=(156.5 \pm 1.5) \mathrm{MeV}$. This average is in agreement with previous results $[5,6,7]$ 

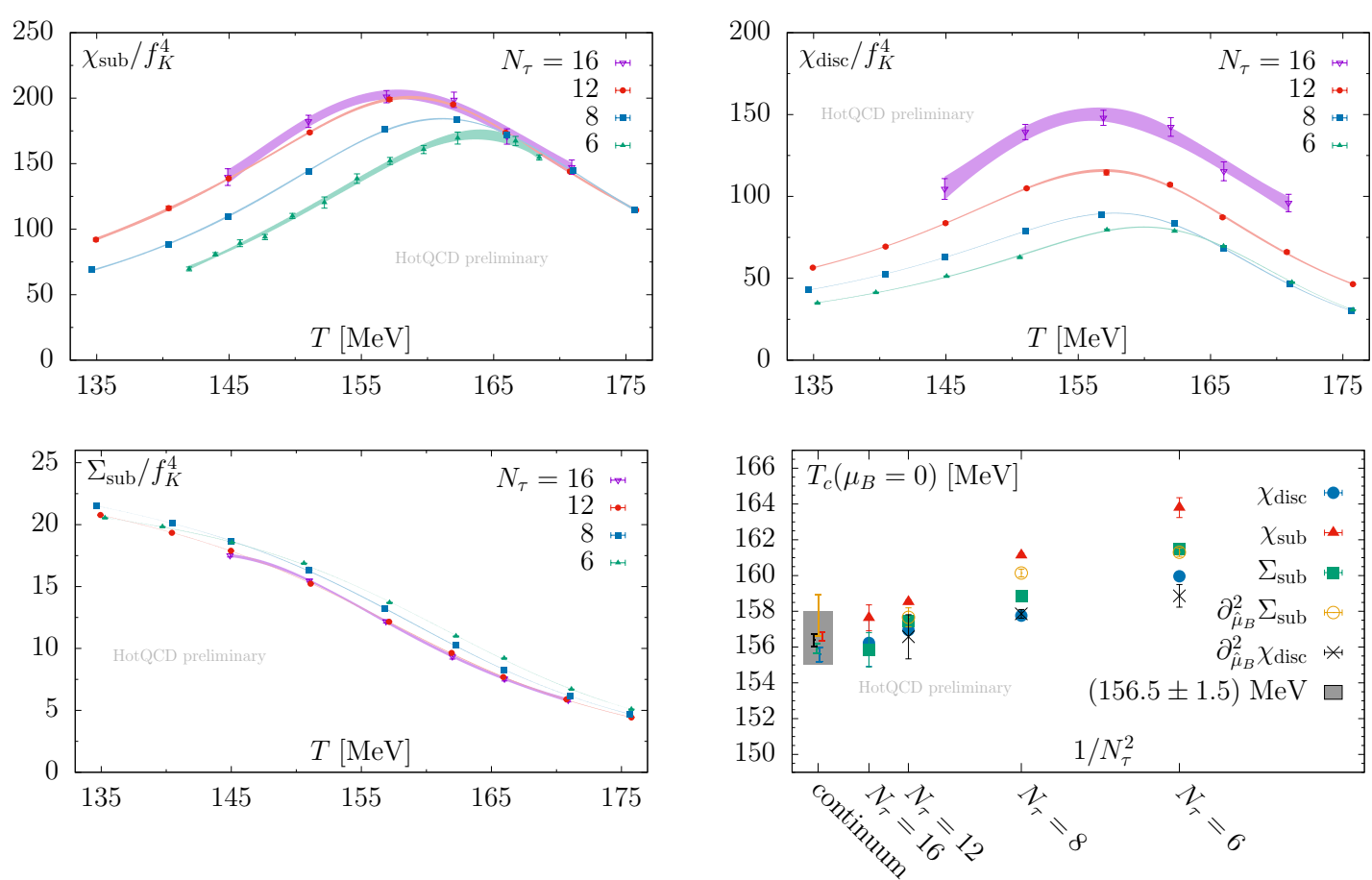

Figure 1: The subtracted chiral susceptibility (top left), the disconnected chiral susceptibility (top right) and the subtracted chiral condensate (bottom left) as a function of the temperature for different $N_{\tau}$. The data is plotted in two-flavor formulation and normalized using the kaon decay constant $f_{K}$. The colored bands are given by AIC weighted Padé approximations which include statistical and systematic errors. In the bottom right plot, we show the obtained chiral crossover temperatures $T_{c}\left(\mu_{B}=0\right)$ as a function of $1 / N_{\tau}^{2}$ for the subtracted condensate $\Sigma_{\text {sub }}\left(T_{c}\right.$ defined by inflection point), the subtracted susceptibility $\chi_{\text {sub }}\left(T_{c}\right.$ defined by maximum), the disconnected susceptibility $\chi_{\text {disc }}\left(T_{c}\right.$ defined by maximum), the second $\hat{\mu}_{B}$ derivative of $\Sigma_{\text {sub }}$ ( $T_{c}$ defined by minimum) and the second $\hat{\mu}_{B}$ derivative of $\chi_{\text {disc }}\left(T_{c}\right.$ defined by zero). All chiral observables define pseudo-critical temperatures. The combined continuum value $(156.5 \pm 1.5) \mathrm{MeV}$ in the gray box is an unweighted average of all observables which includes a $1 \mathrm{MeV}$ error for setting the scale. This combined value resembles systematic effects (ambiguity in defining a pseudo-critical temperature), statistical and scale setting errors.

obtained with different lattice formulations. The curvature coefficients $\kappa_{n}$ can be obtained by requiring that e.g. each order $\mu_{B}^{n}$ in $d / d T\left(\chi_{\text {disc }}\left(T, \hat{\mu}_{B}\right) / f_{K}^{4}\right) \equiv 0$ vanishes [3]. In the following, we derive $\kappa_{2}$ from the chiral susceptibility for vanishing $\mu_{Q}$ and $\mu_{S}$. First, we expand the susceptibility in $T$ and $\hat{\mu}_{B}$ around $\left(T_{0}, \hat{\mu}_{B}=0\right)$. The relevant terms for $\kappa_{2}$ are given by

$$
\begin{aligned}
\frac{\chi_{\mathrm{disc}}\left(T, \mu_{B}\right)}{f_{K}^{4}} & =\left.c_{0}^{\chi}\right|_{\left(T_{0}, 0\right)}+\left.\frac{\partial c_{0}^{\chi}}{\partial T}\right|_{\left(T_{0}, 0\right)}\left(T-T_{0}\right)+\left.\frac{1}{2} \frac{\partial^{2} c_{0}^{\chi}}{\partial T^{2}}\right|_{\left(T_{0}, 0\right)}\left(T-T_{0}\right)^{2} \\
& +\left.\frac{1}{2} c_{2}^{\chi}\right|_{\left(T_{0}, 0\right)} \hat{\mu}_{B}^{2}+\left.\frac{1}{2} \frac{\partial c_{2}^{\chi}}{\partial T}\right|_{\left(T_{0}, 0\right)}\left(T-T_{0}\right) \hat{\mu}_{B}^{2}+\ldots
\end{aligned}
$$

with

$$
c_{n}^{\chi}=\left.\frac{\partial \chi_{\mathrm{disc}} / f_{K}^{4}}{\partial \hat{\mu}_{B}^{n}}\right|_{\mu=0} \quad \text { and } \quad \hat{\mu}=\frac{\mu}{T}
$$


Taking into account that the first $T$ derivative of $c_{0}^{\chi}$ vanishes at $T_{0}$ and neglecting terms which have no contribution to order $\mu_{B}^{2}$, we obtain for the $T$ derivative of the Taylor expansion

$$
\begin{aligned}
\frac{d}{d T} \frac{\chi_{\mathrm{disc}}\left(T, \mu_{B}\right)}{f_{K}^{4}} & =\left.\frac{\partial^{2} c_{0}^{\chi}}{\partial T^{2}}\right|_{\left(T_{0}, 0\right)}\left(T-T_{0}\right)-\left.c_{2}^{\chi}\right|_{\left(T_{0}, 0\right)} \frac{\mu_{B}^{2}}{T^{3}}+\left.\frac{1}{2} \frac{\partial c_{2}^{\chi}}{\partial T}\right|_{\left(T_{0}, 0\right)} \hat{\mu}_{B}^{2}+\ldots \\
& =\left(\left.\frac{1}{2} \frac{\partial c_{2}^{\chi}}{\partial T}\right|_{\left(T_{0}, 0\right)} \frac{1}{T_{0}^{2}}-\left.\frac{\partial^{2} c_{0}^{\chi}}{\partial T^{2}}\right|_{\left(T_{0}, 0\right)} \frac{\kappa_{2}}{T_{0}}-\left.c_{2}^{\chi}\right|_{\left(T_{0}, 0\right)} \frac{1}{T_{0}^{3}}\right) \mu_{B}^{2}+\mathscr{O}\left(\mu_{B}^{4}\right) .
\end{aligned}
$$

In the last step, we used that Eq. (2.4) is only equal to zero along the crossover line, i.e. it has to be evaluated at $T=T_{c}\left(\mu_{B}\right)$. By demanding that order $\mathscr{O}\left(\mu_{B}^{2}\right)$ vanishes, we find that

$$
\kappa_{2}^{\chi}=\frac{1}{2 T_{0}^{2}} \frac{\left.T_{0} \frac{\partial c_{2}^{\chi}}{\partial T}\right|_{\left(T_{0}, 0\right)}-\left.2 c_{2}^{\chi}\right|_{\left(T_{0}, 0\right)}}{\left.\frac{\partial^{2} c_{0}^{\chi}}{\partial T^{2}}\right|_{\left(T_{0}, 0\right)}}
$$

Similarly, we can derive $\kappa_{2}$ from the subtracted condensate by requiring that each order $\mu_{B}^{n}$ in $d^{2} / d T^{2}\left(\Sigma_{\text {sub }}\left(T, \hat{\mu}_{B}\right) / f_{K}^{4}\right) \equiv 0$ vanishes. It is given by

$$
\kappa_{2}^{\Sigma}=\frac{1}{2 T_{0}^{3}} \frac{\left.6 c_{2}^{\Sigma}\right|_{\left(T_{0}, 0\right)}-\left.4 T_{0} \frac{\partial c_{2}^{\Sigma}}{\partial T}\right|_{\left(T_{0}, 0\right)}+\left.T_{0}^{2} \frac{\partial^{2} c_{2}^{\Sigma}}{\partial T^{2}}\right|_{\left(T_{0}, 0\right)}}{\left.\frac{\partial^{3} c_{0}^{\Sigma}}{\partial T^{3}}\right|_{\left(T_{0}, 0\right)}}
$$

The $T$ derivatives of $c_{n}^{\chi}$ and $c_{n}^{\Sigma}$ can be obtained analytically by derivating fits of the corresponding coefficient. The next order $\kappa_{4}$ can be derived from the disconnected susceptibility by requiring that $\mathscr{O}\left(\mu_{B}^{4}\right)$ in Eq. (2.4) vanishes. It reads

$$
\kappa_{4}^{\chi}=\frac{1}{24 T_{0}^{2}} \frac{-72 \kappa_{2}^{\chi} c_{2}^{\chi}-4 c_{4}^{\chi}+T_{0}\left[\frac{\partial c_{4}^{\chi}}{\partial T}+12 \kappa_{2}^{\chi}\left(4 \frac{\partial c_{2}^{\chi}}{\partial T}-T_{0} \frac{\partial^{2} c_{2}^{\chi}}{\partial T^{2}}+\kappa_{2}^{\chi} T_{0}^{2} \frac{\partial^{3} c_{0}^{\chi}}{\partial T^{3}}\right)\right]}{\frac{\partial^{2} c_{0}^{\chi}}{\partial T^{2}}}
$$

where all coefficients and their derivatives have to be evaluated at $\left(T_{0}, \hat{\mu}_{B}=0\right)$. For the subtracted condensate the 4 th order curvature coefficient is given by

$$
\begin{aligned}
\kappa_{4}^{\Sigma}= & \frac{288 \kappa_{2}^{\Sigma} c_{2}^{\Sigma}+20 c_{4}^{\Sigma}-216 T_{0} \kappa_{2}^{\Sigma} \frac{\partial c_{2}^{\Sigma}}{\partial T}-8 T_{0} \frac{\partial c_{4}^{\Sigma}}{\partial T}}{\frac{1}{24 T_{0}^{3}} \frac{\partial^{3} c_{0}^{\Sigma}}{\partial T^{3}}} \\
+ & \frac{T_{0}^{2}\left(\frac{\partial^{2} c_{4}^{\Sigma}}{\partial T^{2}}+12 \kappa_{2}^{\Sigma}\left(6 \frac{\partial^{2} c_{2}^{\Sigma}}{\partial T^{2}}-T_{0} \frac{\partial^{3} c_{2}^{\Sigma}}{\partial T^{3}}+\kappa_{2}^{\Sigma} T_{0}^{2} \frac{\partial^{4} c_{0}^{\Sigma}}{\partial T^{4}}\right)\right)}{\frac{1}{24 T_{0}^{3}} \frac{\partial^{3} c_{0}^{\Sigma}}{\partial T^{3}}} .
\end{aligned}
$$

The coefficients $c_{n}^{\chi}$ and $c_{n}^{\Sigma}$ have been derived in [3]. In Fig. 2, we compare the parameterization of the crossover line with results on chemical freeze-out temperatures extracted from heavy-ion collision experiments such as ALICE [9] and STAR [10]. The mean of the ALICE freeze-out temperature of $156.5 \pm 1.5 \mathrm{MeV}$ agrees with our crossover line at almost vanishing baryon chemical potential. The STAR data seems to extrapolate to a significantly higher freeze-out temperature resulting in values well above our crossover line which suggests that both cannot hold simultaneously. 

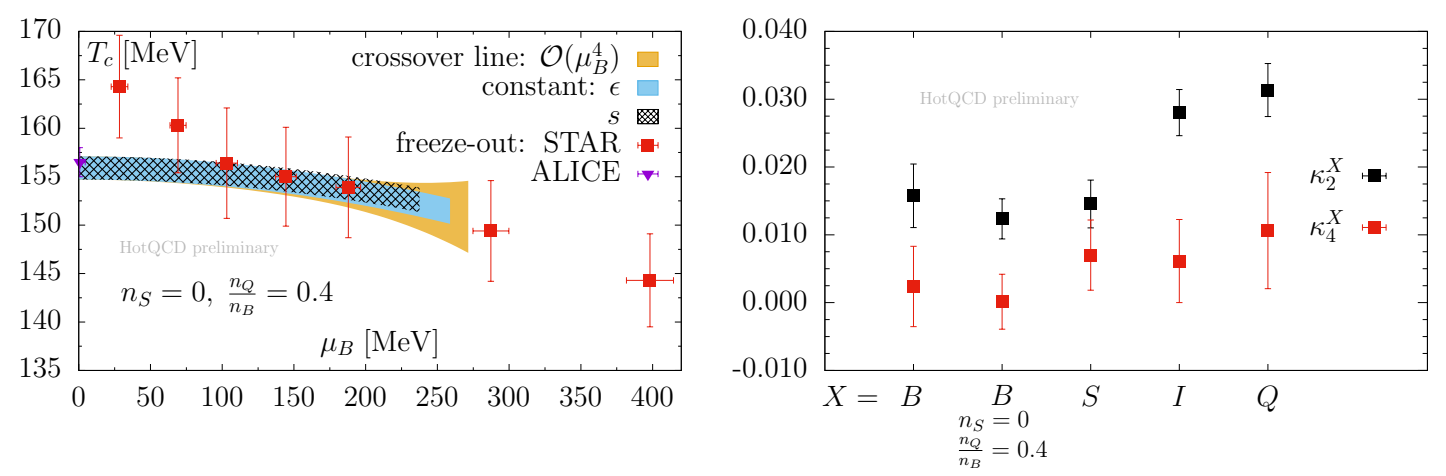

Figure 2: The crossover temperature $T_{C}\left(\mu_{B}\right)$ (left) as a function of the baryon chemical potential $\mu_{B}$ for a strangeness neutral system with $n_{Q} / n_{B}=0.4$ including continuum extrapolated corrections (yellow band) up to $\mathscr{O}\left(\mu_{B}^{4}\right)$. Here, $n_{Q}$ is the mean electric charge density and $n_{B}$ the mean net baryon-number density. All required expansion coefficients $\kappa_{n}$ have been determined from the subtracted condensate $\Sigma_{\text {sub }}$. On top of it, we show lines of constant physics for energy density $\varepsilon$ and entropy density $s$ taken from [8]. The data points represent chemical freeze-out parameters extracted from the ALICE [9] and STAR [10] experiments. The right figure compares the crossover curvature coefficients $\kappa_{2}$ and $\kappa_{4}$ for systems with different constrains. Here, the crossover line is defined as $T_{c}\left(\mu_{X}\right) / T_{0}=1-\kappa_{2}^{X}\left(\mu_{X} / T_{0}\right)^{2}-\kappa_{4}^{X}\left(\mu_{X} / T_{0}\right)^{4}+\mathscr{O}\left(\mu_{X}^{6}\right)$ where $T_{0}$ is the crossover temperature at zero chemical potentials and $X$ is a placeholder for baryon-number $B$, electric charge $Q$, strangeness $S$ and isospin $I$. We also show results of the curvature along $\mu_{B}$ with the constrains $n_{S}=0$ and $n_{Q} / n_{B}=0.4$. The coefficients have been determined from a Taylor expansion of $\chi_{\text {disc }}$. Extracting these coefficients from $\Sigma_{\text {sub }}$ gives similar results. The values are listed in [3].

However, previous results $[5,6,7,11]$ report curvatures which are in agreement with our crossover line. Additionally, we compare the crossover line to lines of constant physics (LCPs) from lattice QCD simulations [8]. The LCP curvatures from energy density and entropy density agree with the crossover curvature within errors. Furthermore, we explored the crossover along several directions and for different constrains in the QCD phase diagram. We found that the QCD phase diagram has very similar curvatures $\kappa_{2}$ in all directions except along directions of non-zero electric charge chemical potential $\mu_{Q}$ and isospin chemical potential $\mu_{I}$. In these cases, the curvature $\kappa_{2}$ is two times larger (see Fig. 2).

\section{Fluctuations along the QCD crossover}

In the following, we study fluctuations of net baryon-number given by

$$
\sigma_{B}^{2}=\frac{\partial \ln Z}{\partial \hat{\mu}_{B}^{2}}
$$

It has been shown successfully [12] that net baryon-number fluctuations couple to the condensate and thus would reveal critical behavior when approaching a critical point. Particularly interesting is to study their deviations from the HRG model. Even for a finite volume, as given in heavy-ion collisions, these fluctuations should resemble some critical behavior in the vicinity of a critical point, i.e. show substantially larger fluctuations compared to a HRG. The relative change of $\sigma_{B}^{2}$ can 

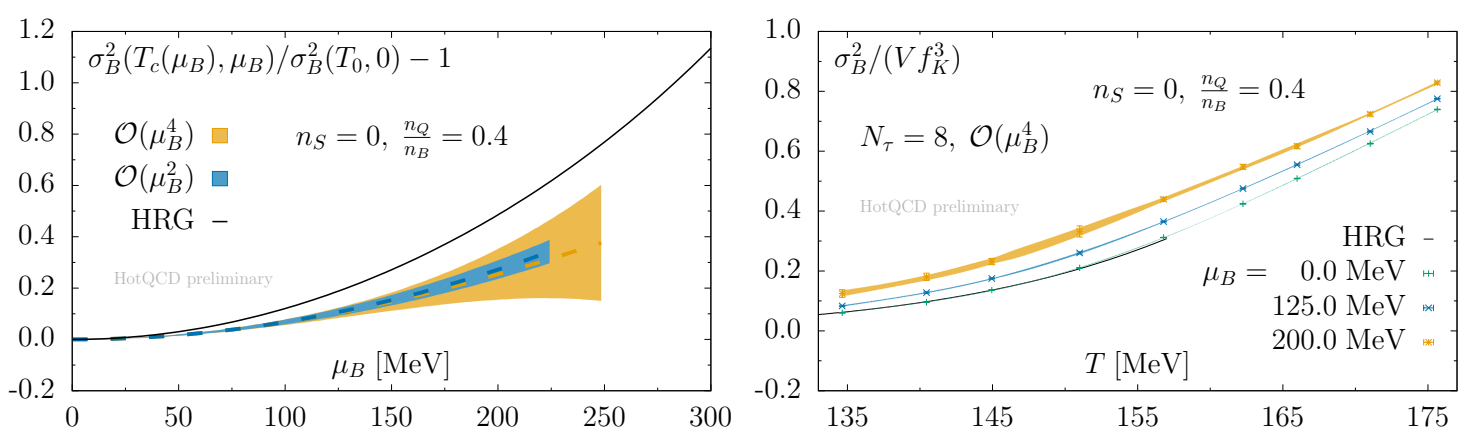

Figure 3: The relative change of net baryon-number fluctuations $\sigma_{B}^{2}$ (left) along the crossover line $T_{c}\left(\mu_{B}\right)$ as a function of $\mu_{B}$ for a system with strangeness neutrality and $n_{Q} / n_{B}=0.4$. Here, the curvature of $T_{c}\left(\mu_{B}\right)$ has been determined from the subtracted chiral condensate $\Sigma_{\text {sub. The blue band includes continuum extrapolated }}$ corrections up to $\mathscr{O}\left(\mu_{B}^{2}\right)$ and the yellow band up to $\mathscr{O}\left(\mu_{B}^{4}\right)$. The corresponding mean is visualized using a dashed line. Also shown are HRG results using a solid black line evaluated on along a curvature defined by the mean of $T_{c}\left(\mu_{B}\right)$. In the right figure, we show $\sigma_{B}^{2}$ as function of the temperature at three values of baryon chemical potential $\mu_{B}$ for a finite lattice with $N_{\tau}=8$ including corrections up to $\mathscr{O}\left(\mu_{B}^{4}\right)$. For vanishing baryon chemical potential, we compare QCD results to the HRG as shown by a solid black line.
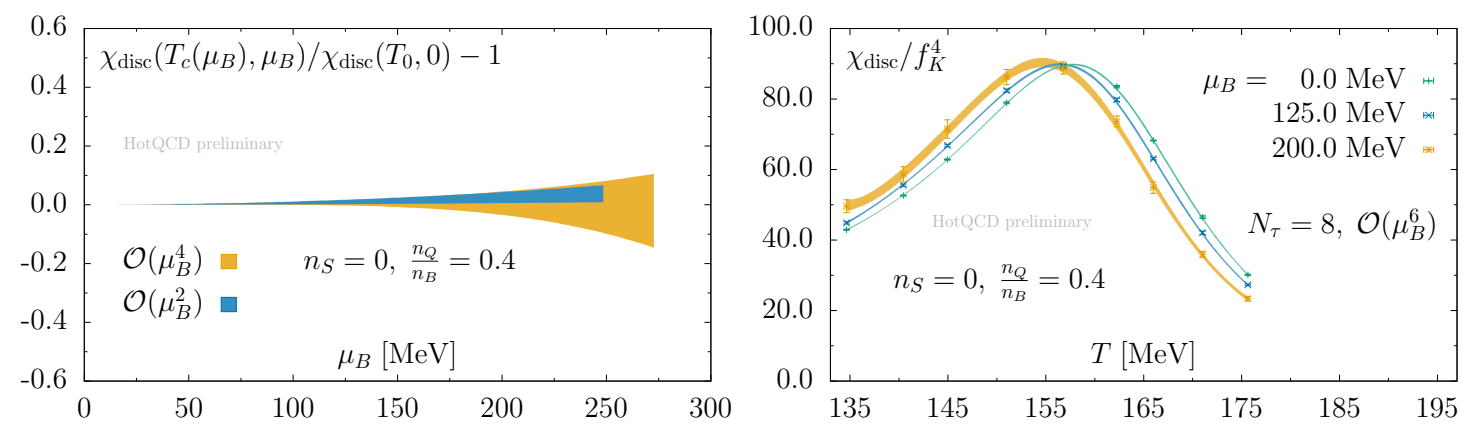

Figure 4: The relative change of the disconnected chiral susceptibility $\chi_{\text {disc }}$ (left) along the crossover line as a function of $\mu_{B}$ for a system with strangeness neutrality and $n_{Q} / n_{B}=0.4$. Here, the curvature of $T_{c}\left(\mu_{B}\right)$ has been determined from $\chi_{\text {disc }}$. The blue band includes continuum extrapolated corrections up to $\mathscr{O}\left(\mu_{B}^{2}\right)$ and the yellow band up to $\mathscr{O}\left(\mu_{B}^{4}\right)$. In the right figure, we show $\chi_{\text {disc }}$ as function of the temperature at three values of baryon chemical potential $\mu_{B}$ for a finite lattice with $N_{\tau}=8$ including corrections up to $\mathscr{O}\left(\mu_{B}^{6}\right)$.

be expressed in a Taylor series

$$
\frac{\sigma_{B}^{2}\left(T_{c}\left(\mu_{B}\right), \mu_{B}\right)-\sigma_{B}^{2}\left(T_{0}, 0\right)}{\sigma_{B}^{2}\left(T_{0}, 0\right)}=\lambda_{2}\left(\frac{\mu_{B}}{T_{0}}\right)^{2}+\lambda_{4}\left(\frac{\mu_{B}}{T_{0}}\right)^{4}+\mathscr{O}\left(\mu_{B}^{6}\right)
$$

where the expansion coefficients $\lambda_{n}$ can be determined using lattice QCD. We have continuum extrapolated these coefficients up to $\mathscr{O}\left(\mu_{B}^{4}\right)$ which are used in Fig. 3 to visualize the relative change along $T_{c}\left(\mu_{B}\right)$. For the strangeness neutral case, the fluctuations are at least a factor two smaller compared to a HRG. Given that Taylor expansions for baryon-number fluctuations in the HRG model have an infinite radius of convergence and substantially larger fluctuations compared to our lattice results, we conclude that it is unlikely that a QCD critical point can be found for $\mu_{B}$ smaller than $250 \mathrm{MeV}$ along the crossover line. Similarly, we have studied chiral susceptibility fluctuations 
along the crossover line. As can be seen from Fig. 4, this analysis shows a constant peak height for $\chi_{\text {disc }}$, i.e. no significant change along the crossover line. This suggests that for $\mu_{B}<250 \mathrm{MeV}$ no signs for a narrowing of the crossover region or increasing correlation length have been observed. In addition, we also measured 6th order expansion coefficients for a fixed lattice spacing and found that these higher order corrections are negligible for $\mu_{B}<250 \mathrm{MeV}$ for $\chi_{\text {disc }}$ and $\sigma_{B}^{2}$ along the crossover line.

Acknowledgments This work was supported through Contract No. DE-SC001270 with the U.S. Department of Energy and through the grant CRC-TR 211 "Strong-interaction matter under extreme conditions" with the Deutsche Forschungsgemeinschaft (DFG). Numerical calculations have been made possible through computing resources granted by ALCC, INCITE, NESAP, PRACE and USQCD.

\section{References}

[1] Y. Aoki, et al., Nature 443 (2006) 675-678. arXiv: hep-lat / 0611014.

[2] T. Bhattacharya, et al., Phys. Rev. Lett. 113 (8) (2014) 082001. arXiv:1402 . 5175.

[3] P. Steinbrecher, pub.uni-bielefeld.de/publication/2919977 (2018).

[4] A. Bazavov, et al., Phys. Rev. D 85 (2012) 054503. arXiv:1111. 1710.

[5] C. Bonati, et al., Phys. Rev. D92 (5) (2015) 054503. arXiv:1507. 03571.

[6] C. Bonati, et al., (2018). arXiv:1805.02960.

[7] R. Bellwied, et al., Phys.Lett. B 751 (2015) 559-564. arXiv:1507. 07510.

[8] A. Bazavov, et al., Phys.Rev. D 95 (2017) 054504. arXiv: 1701.04325.

[9] A. Andronic, et al., Nature 561 (7723) (2018) 321-330. arXiv:1710.09425.

[10] STAR Collaboration, Phys.Rev. C 96 (2017) 044904. arXiv: 1701.07065.

[11] P. Cea, L. Cosmai, A. Papa, Phys. Rev. D93 (1) (2016) 014507. arXiv:1508. 07599.

[12] Y. Hatta, M. A. Stephanov, Phys. Rev. Lett. 91 (2003) 102003. arXiv: hep-ph/0302002. 